Risk Attitudes and Decision Weights

Author(s): Amos Tversky and Peter Wakker

Source: Econometrica, Vol. 63, No. 6 (Nov., 1995), pp. 1255-1280

Published by: The Econometric Society

Stable URL: http://www.jstor.org/stable/2171769

Accessed: $27 / 04 / 2011$ 08:19

Your use of the JSTOR archive indicates your acceptance of JSTOR's Terms and Conditions of Use, available at http://www.jstor.org/page/info/about/policies/terms.jsp. JSTOR's Terms and Conditions of Use provides, in part, that unless you have obtained prior permission, you may not download an entire issue of a journal or multiple copies of articles, and you may use content in the JSTOR archive only for your personal, non-commercial use.

Please contact the publisher regarding any further use of this work. Publisher contact information may be obtained at http://www.jstor.org/action/showPublisher?publisherCode=econosoc.

Each copy of any part of a JSTOR transmission must contain the same copyright notice that appears on the screen or printed page of such transmission.

JSTOR is a not-for-profit service that helps scholars, researchers, and students discover, use, and build upon a wide range of content in a trusted digital archive. We use information technology and tools to increase productivity and facilitate new forms of scholarship. For more information about JSTOR, please contact support@jstor.org. 


\title{
RISK ATTITUDES AND DECISION WEIGHTS ${ }^{1}$
}

\author{
By Amos Tversky and Peter Wakker
}

\begin{abstract}
To accommodate the observed pattern of risk-aversion and risk-seeking, as well as common violations of expected utility (e.g., the certainty effect), we introduce and characterize a weighting function according to which an event has greater impact when it turns impossibility into possibility, or possibility into certainty, than when it merely makes a possibility more or less likely. We show how to compare such weighting functions (of different individuals) with respect to the degree of departure from expected utility, and we present a method for comparing an individual's weighting functions for risk and for uncertainty.
\end{abstract}

KEYWORDS: Risk attitude, decision weights, rank dependence, source dependence.

\section{INTRODUCTION}

THE CLASSICAL THEORY OF DECISION under risk and uncertainty combines the principle of mathematical expectation with the assumption of decreasing marginal utility, which jointly imply risk aversion. Three clusters of phenomena reflecting risk attitudes have challenged the descriptive validity of the classical theory. First, although risk aversion is prevalent, there are situations in which risk seeking is commonly observed. Gambling is a case in point. Second, there is a considerable body of evidence that preferences between risky prospects are not linear in the probabilities. The certainty effect, demonstrated by Allais, is the best-known example of this phenomenon. Third, people's preferences depend not only on the degree of uncertainty but also on the source of uncertainty. For instance, people sometimes prefer to bet on known rather than unknown probabilities, as demonstrated by Ellsberg.

There have been many attempts to explain risk attitudes that are inconsistent with expected utility. Much recent work has been devoted to theories that extend expected utility by introducing nonadditive decision weights (Kahneman and Tversky (1979), Quiggin (1982), Yaari (1987), Gilboa (1987), Schmeidler (1989), Luce and Fishburn (1991)). In these models, preferences are determined jointly by the utility function that measures the subjective value of the outcomes, and by the decision weights that capture what may be called chance attitude.

In this article we present a theoretical analysis of decision weights that is motivated by the observed pattern of risk seeking, nonlinear preferences, and source dependence. This pattern suggests an $S$-shaped weighting function that overweights small probabilities and underweights moderate and high probabilities (Section 2). The theoretical framework used in this paper is introduced in Section 3. Section 4 establishes the properties of the preference order that are necessary and sufficient for an $S$-shaped weighting function. This analysis is

\footnotetext{
${ }^{1}$ This research was supported by Grant SES-9109535 from the National Science Foundation.
} 
extended to uncertainty in Section 5. In analogy to the Pratt/Arrow analysis of comparative risk aversion, Section 6 introduces the relation more-SA-than between the weighting functions of different individuals, which reflects departure from expected utility. Section 7 introduces a method for comparing the weighting function of the same individual for different sources of uncertainty. This method is used to analyze the observed relation between risk and uncertainty. Proofs are deferred to the Appendix.

\section{THE FOURFOLD PATTERN}

In order to motivate the present development, we first illustrate some common features of people's attitude toward risk. Consider simple prospects of the form $(x, p)$ that offer $\$ x$ with probability $p$, and nothing otherwise. The study of choice between simple risky prospects has given rise to the fourfold pattern illustrated in Table I. These data are taken from a study by Tversky and Kahneman (1992) in which each subject made a series of choices between a risky prospect and various cash offers. The value of $C(x, p)$ is the median cash offer (in dollars) that was indifferent to the prospect $(x, p)$.

Table I exhibits risk seeking for gains and risk aversion for losses of low probability combined with risk aversion for gains and risk seeking for losses of high probability. This pattern has been observed in numerous studies, with and without contingent payoffs (Fishburn and Kochenberger (1979), Kahneman and Tversky (1979), Hershey and Schoemaker (1980), Payne, Laughhunn, and Crum (1981), Cohen, Jaffray, and Said (1987), Wehrung (1989), Tversky and Kahneman (1992)). Extreme risk seeking for long shots has recently been reported by Kachelmeier and Shehata (1992) in an experiment conducted in China with real payoffs that were considerably higher than the subjects' normal monthly income. Risk seeking for small probabilities of gains is consistent with common observations of gambling and risky ventures, whereas risk seeking for high-probability losses is consistent with the tendency to accept a risk in order to avoid a sure loss.

Friedman and Savage (1948) and Markowitz (1952) have attempted to explain the combination of risk seeking and risk aversion in terms of a utility function with both concave and convex regions. However, because the fourfold pattern arises over a wide range of payoffs, it cannot be explained by the utility function for money. Instead, it suggests a nonlinear transformation of the probability scale.

TABLE I

The Fourfold Pattern of Risk Attitudes

\begin{tabular}{lcc}
\hline & Gain & Loss \\
\hline Low probability & $C(100, .05)=14$ & $C(-100, .05)=-8$ \\
(Risk Seeking) & (Risk Aversion) \\
High probability & $\begin{array}{l}C(100, .95)=78 \\
\text { (Risk Aversion) }\end{array}$ & $\begin{array}{c}C(-100, .95)=-84 \\
\text { (Risk Seeking) }\end{array}$ \\
\hline
\end{tabular}


Suppose the value of the prospect $(x, p)$ is given by $w(p) v(x)$, where $v$ is the value function for gains and losses, and $w$ is a nonlinear weighting function. Figure 1 presents a typical weighting function obtained by Tversky and Fox (1994). This function exhibits diminishing sensitivity: it is steepest near the endpoints and shallower in the middle, yielding overweighting of small probabilities and underweighting of middle and high probabilities. Thus, people underestimate the impact of an increase in probability from $20 \%$ to $25 \%$ in comparison to an increase from $0 \%$ to $5 \%$ or from $95 \%$ to $100 \%$. Such a weighting function gives rise to the fourfold pattern described above, under plausible assumptions concerning the value function.

\section{BASIC CONCEPTS}

We first introduce terminology and notation, and then describe the theoretical framework used in the paper. We distinguish decision under risk, where the probabilities are assumed to be known, and decision under uncertainty, where the probabilities associated with the various outcomes are not given in advance.

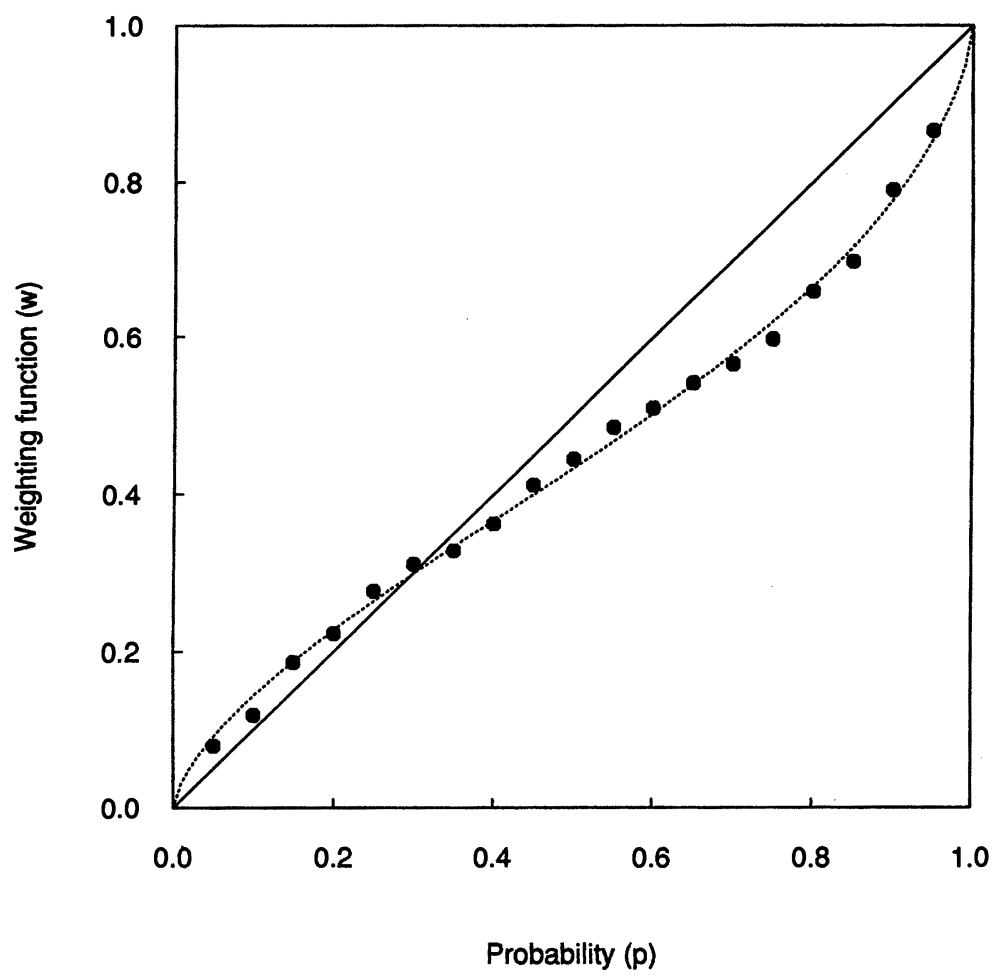

FIGURE 1.-The points represent median estimates, across subjects $(N=40)$, obtained in Tversky and Fox (1994). The smooth curve is obtained by fitting the parametric form $w(p)=\delta p^{\gamma} /\left(\delta p^{\gamma}+\right.$ $\left.(1-p)^{\gamma}\right)$, suggested by Lattimore, Baker, and Witte (1992). The estimated values of the parameters are $\gamma=.69, \delta=.77$. 
In both cases, the decision maker has to select between prospects that are described as positive or negative changes with respect to the status quo. To simplify matters, we assume that the outcomes are real numbers designating money, and interpret 0 as the status quo. Gains refer to positive outcomes, and losses refer to negative outcomes. In decision under risk a prospect is described by a finite probability distribution. Thus $\left(x_{1}, p_{1} ; \ldots ; x_{n}, p_{n}\right)$ is the risky prospect yielding outcome $x_{j}$ with probability $p_{j}, j=1, \ldots, n$; the $p_{j}$ 's are nonnegative and sum to one. If there is only one nonzero outcome then the zero outcome is suppressed; for example, $(Z, 1 / 2)$ is the prospect that yields $Z$ with probability $1 / 2$ and 0 with probability $1 / 2$.

Decision under uncertainty is described in terms of a set $S$, called the state space. We assume that exactly one state obtains, but the decision maker is uncertain about this state. Subsets of $S$ are called events; $S-A$ is the complement to $A$. In decision under uncertainty, prospects are functions from $S$ to $\mathbb{R}$, taking finitely many values. If state $s$ obtains, then prospect $f$ yields the outcome $f(s)$. An uncertain prospect is described as $\left(x_{1}, A_{1} ; \ldots ; x_{n}, A_{n}\right)$, where $\left(A_{1}, \ldots, A_{n}\right)$ is a partition of $S$ and $x_{j}$ is the outcome associated with the states in $A_{j}$. As above, the zero outcome is suppressed if there is only one nonzero outcome; thus $(x, A)$ is the prospect that yields $x$ if $A$ obtains, and 0 if it does not.

Risk can be considered as a special case of uncertainty where probabilities are given for the events in $S$, and prospects that generate the same probability distribution over the outcomes are treated as identical. In this case, each prospect is described by the probability distribution it induces over the outcomes, with no reference to the state space.

We identify outcomes with degenerate prospects. Thus $x$ can be viewed as a constant function assigning outcome $x$ to all states or as a degenerate probability distribution assigning probability 1 to that outcome. Let $\succcurlyeq$ denote the preference relation over prospects; the relations $\succ, \sim$ are defined as usual.

\section{Cumulative Prospect Theory}

This article adopts the theoretical framework of cumulative prospect theory, or CPT for short (Tversky and Kahneman (1992)). This theory is more general than the rank-dependent utility model because it permits a different treatment of gains and losses. ${ }^{2}$ It assumes a continuous strictly increasing value function $v$ : $\mathbb{R} \rightarrow \mathbb{R}$ satisfying $v(0)=0$. For choice under risk, it invokes two weighting functions, denoted by $w^{+}$and $w^{-}$, for gains and losses respectively. ${ }^{3} \mathrm{~A}$ weighting function $w$ is a strictly increasing function from $[0,1]$ to $[0,1]$ with $w(0)=0$ and $w(1)=1$. For uncertainty, the weighting functions for gains and losses are denoted by $W^{+}$and $W^{-}$. Here a weighting function (or a capacity) $W$ on $S$ is a

\footnotetext{
${ }^{2}$ Closely related models were proposed by Starmer and Sugden (1989) and Luce and Fishburn (1991).

${ }^{3}$ In following sections the superscript + is often suppressed.
} 
function on $2^{S}$ such that $W(\varnothing)=0, W(S)=1$, and $W(A) \geq W(B)$ whenever $A \supset B$. Obviously, if $W$ is additive, i.e., $W(A \cup B)=W(A)+W(B)$ for all disjoint events $A, B$, then it is a probability measure.

According to CPT, the value of a prospect $\left(x_{1}, p_{1} ; \ldots ; x_{n}, p_{n}\right)$ in which $x_{1} \leq \cdots \leq x_{k} \leq 0 \leq x_{k+1} \leq \cdots \leq x_{n}$, is

$$
\sum_{j=1}^{k} \pi_{j}^{-} v\left(x_{j}\right)+\sum_{j=k+1}^{n} \pi_{j}^{+} v\left(x_{j}\right),
$$

where the decision weights are defined by $\pi_{j}^{-}=w^{-}\left(p_{1}+\cdots+p_{j}\right)-w^{-}\left(p_{1}\right.$ $\left.+\cdots+p_{j-1}\right)$ and $\pi_{j}^{+}=w^{+}\left(p_{j}+\cdots+p_{n}\right)-w^{+}\left(p_{j+1}+\cdots+p_{n}\right){ }^{4}$ Note that these weights do not necessarily sum to one. For uncertainty, the value of a prospect $\left(x_{1}, A_{1} ; \ldots ; x_{n}, A_{n}\right)$, in which $x_{1} \leq \cdots \leq x_{k} \leq 0 \leq x_{k+1} \leq \cdots \leq x_{n}$, is

$$
\sum_{j=1}^{k} \pi_{j}^{-} v\left(x_{j}\right)+\sum_{j=k+1}^{n} \pi_{j}^{+} v\left(x_{j}\right),
$$

where now decision weights are defined by $\pi_{j}^{-}=W^{-}\left(A_{1} \cup \cdots \cup A_{j}\right)-W^{-}\left(A_{1}\right.$ $\left.\cup \cdots \cup A_{j-1}\right)$ and $\pi_{j}^{+}=W^{+}\left(A_{j} \cup \cdots \cup A_{n}\right)-W^{+}\left(A_{j+1} \cup \cdots \cup A_{n}\right)^{5}$

CPT generalizes rank-dependent utility, introduced by Quiggin (1982) and Yaari (1987) in the context of risk, and by Schmeidler (1989) in the context of uncertainty. Rank-dependent utility corresponds to the special case where the weighting function for losses is the dual of the weighting function for gains, i.e., $w^{-}(p)=1-w^{+}(1-p)$, and $W^{-}(A)=1-W^{+}(S-A)$. For prospects with nonnegative outcomes, rank-dependent utility coincides with CPT. Tversky and Kahneman (1992) considered another special case of CPT, where $w^{+}=w^{-}$or $W^{+}=W^{-}$, which provided a reasonably good fit for risk choice. Preference conditions for this property, called reflection, are presented in Appendix B.

We assume that the weighting function for risk is continuous, and that the weighting function for uncertainty satisfies solvability, ${ }^{6}$ i.e., for all events $A \subset C$ and $W(A) \leq p \leq W(C)$ there exists an event $B$ such that $W(B)=p$ and $A \subset B \subset$ $C$. The assumptions made throughout this paper are summarized below; they have been axiomatized in Wakker and Tversky (1993, Section 8.4):

AssumPTION 3.1: Risky prospects are probability distributions over $\mathbb{R}$. Uncertain prospects are functions from the state space $S$ to the outcome set $\mathbb{R}$. Prospects have finitely many outcomes. Preferences between prospects are represented by (3.1) or (3.2). The value function is continuous and strictly increasing. The weighting function for risk is continuous and strictly increasing; for uncertainty it satisfies solvability.

\footnotetext{
${ }^{4}$ Here we follow the usual convention that, for $j=0, p_{1}+\cdots+p_{j}=0$, and for $j=n, p_{j+1}$ $+\cdots+p_{n}=0$.

${ }^{5}$ For $j=0, A_{1} \cup \cdots \cup A_{j}=\varnothing$; for $j=n, A_{j+1} \cup \cdots \cup A_{n}=\varnothing$.

${ }^{6}$ Gilboa (1987) introduced this condition under the name convex-ranged.
} 


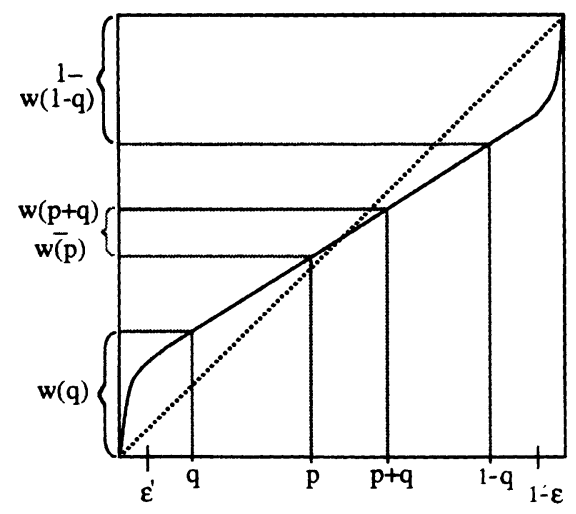

FIGURE 2.-Illustration of SA.

\section{SUBADDITIVITY IN CHOICE UNDER RISK}

In this article we focus on the weighting function for gains. The analysis for losses is essentially identical and, with few exceptions, will not be discussed separately. Thus we restrict attention to nonnegative outcomes, and suppress the superscript in $w^{+}$and $W^{+}$. This section discusses risk; uncertainty is discussed in following sections.

The weighting function presented in Figure 1 is $S$-shaped: It is steepest near 0 and 1 and shallower in the middle. The experimental evidence is generally consistent with such a weighting function (Camerer (1992), Cohen and Jaffray (1988), Tversky and Kahneman (1992)). Thus, in Figure 1 a "lower" interval $[0, q]$ has more impact than a middle interval $[p, p+q]$ provided the middle interval is bounded away from the upper endpoint 1 (e.g., $p+q \leq .9$ ). Similarly, an "upper" interval $[1-q, 1]$ has more impact than a middle interval $[p, p+q]$ provided the middle interval is bounded away from the lower endpoint 0 (e.g., $p \geq .1$ ). The following definition formalizes this notion (see Figure 2): $w$ satisfies bounded subadditivity, or subadditivity $(S A)$ for short, ${ }^{7}$ if there exist constants $\varepsilon \geq 0$ and $\varepsilon^{\prime} \geq 0$ such that

$$
w(q) \geq w(p+q)-w(p) \text { whenever } p+q \leq 1-\varepsilon
$$

and

$$
1-w(1-q) \geq w(p+q)-w(p) \text { whenever } p \geq \varepsilon^{\prime} .
$$

Conditions (4.1) and (4.2) are called lower $S A$ and upper $S A$, respectively. Lower SA entails the inequality $w(p+q) \leq w(p)+w(q)$ on the interval $[0,1-\varepsilon]$. Upper SA implies the same inequality on the interval $\left[0,1-\varepsilon^{\prime}\right]$ for the dual weighting function $\hat{w}(p)=1-w(1-p)$, as can be seen by substitution. The constants $\varepsilon, \varepsilon^{\prime}$ are called boundary constants, and do not depend on $p, q$. They serve to ensure that we always compare an interval that includes 0 or 1 with an

\footnotetext{
${ }^{7}$ For convenience, we use the terms bounded SA or SA instead of the more accurate term $\varepsilon, \varepsilon^{\prime}-\mathrm{SA}$.
} 
interval that does not. Naturally, they may vary from one individual to the other. We are primarily interested in lower SA near 0 , say on the interval $[0,0.4]$, and in upper SA near 1 , say on the interval $[0.6,1]$. This corresponds to $\varepsilon=\varepsilon^{\prime}=.6$. Since $w$ is fairly linear in the middle region, lower and upper SA usually hold for larger intervals, and for most functions found in the literature $\varepsilon=.1$ and even $\varepsilon^{\prime}=0$ can be chosen. For instance, for the weighting function depicted in Figure 1, SA holds for boundary constants $\varepsilon=.07$ and $\varepsilon^{\prime}=0$, and hence for any larger boundary constants.

\section{Preference Conditions}

Next we present conditions for preferences that are necessary and sufficient for bounded subadditivity. These conditions are independent of the value function, and thus separate what we have called chance attitude from marginal utility. Previous work assumed a linear value function (Yaari (1987), Chateauneuf (1991)) or a concave value function (Chew, Karni, and Safra (1989), Chew (1989)). Furthermore these papers investigated convex, rather than subadditive, weighting functions.

We begin with the certainty effect, which leads to upper SA (4.2). As demonstrated by Allais, people commonly exhibit the following preferences, where $M$ denotes one million dollar:

$$
\begin{aligned}
(1 \mathrm{M}, .11) & \prec(5 \mathrm{M}, .10) \quad \text { and } \\
1 \mathrm{M} & \succ(0, .01 ; 1 \mathrm{M}, .89 ; 5 \mathrm{M}, .10) .
\end{aligned}
$$

The certainty effect suggests the preference condition that is needed to characterize upper SA. To illustrate, let us shift probability mass from $5 \mathrm{M}$ to 0 in the upper right prospect until the decision maker is indifferent between the upper prospects. Suppose we find

$$
(1 \mathrm{M}, .11) \sim(5 \mathrm{M} ., .08) \text {. }
$$

Obviously, from the second preference above it follows by dominance that the same probability shift (.2 from $5 \mathrm{M}$ to 0 on the right) yields

$$
1 M \succ(0, .03 ; 1 \mathrm{M}, .89 ; 5 \mathrm{M}, .08) \text {. }
$$

In general, upper $\mathrm{SA}$ requires that

$$
\begin{aligned}
(z, 1-q) & \sim(Z, p) \quad \text { implies } \\
z & \geqslant(0,1-p-q ; z, q ; Z, p)
\end{aligned}
$$

for $0<z<Z$, and $p \geq \varepsilon^{\prime}$ where $\varepsilon^{\prime} \geq 0$ is the boundary constant. ${ }^{8}$ To interpret the condition, recall that in CPT prospects are evaluated in terms of cumulative events, e.g., receiving $z$ or more. According to (4.3), an increase of $q$ in the probability of that event has more impact on the left, where it makes that event certain than on the right where it merely makes the event more probable. It is instructive to note that the CPT difference between the left prospects in (4.3) is

\footnotetext{
${ }^{8}$ Segal (1987) proposed a similar generalization of the Allais example which implies a convex weighting function.
} 
$(1-w(1-q)) v(z)$, and between the right prospects is $(w(p+q)-w(p)) v(z)$; hence (4.2) implies (4.3) since $v(z)>0$.

Next we turn to the overweighting of small probabilities. Consider the following preferences.

$$
\begin{aligned}
40 & \prec(0, .35 ; 40, .05 ; 100, .60) \\
(40, .95 ; 100, .05) & \succ(100, .65) .
\end{aligned}
$$

Let us shift probability mass from $\$ 100$ to 0 in the upper right prospect until the decision maker is indifferent between the upper prospects. Suppose we find

$$
40 \sim(0, .45 ; 40, .05 ; 100, .50) .
$$

From the second preference above it follows by dominance that the same probability shift (.10 from 100 to 0 on the right) yields

$$
(40, .95 ; 100, .05) \succ(100, .55) \text {. }
$$

In general, lower SA requires that

$$
\begin{aligned}
z & \sim(0,1-p-q ; z, q ; Z, p) \text { implies } \\
(z, 1-q ; Z, q) & \geqslant(Z, p+q),
\end{aligned}
$$

for $0<z<Z$ and $p+q \leq 1-\varepsilon$, where $\varepsilon \geq 0$ is the boundary constant. Thus a $q$ probability shift from $z$ to $Z$ has more impact on the left, where it makes the receipt of (at least) $Z$ possible, than on the right where it merely increases the probability of receiving (at least) $Z$. Note that for the right prospects the extreme outcomes, 0 and $Z$, did not change, whereas for the left prospects the best outcome changed from $z$ to $Z$. To see that lower SA implies (4.4), note that the CPT difference between the left prospects in (4.4) is $w(q)(v(Z)-v(z))$, and between the right prospects it is $(w(p+q)-w(p))(v(Z)-v(z))$.

Proposition 4.1: Under Assumption 3.1, the weighting function $w$ satisfies $S A$ if and only if the preference relation satisfies (4.3) and (4.4). ${ }^{9}$

Actually, lower SA is equivalent to (4.4), and upper SA to (4.3).

\section{Applications}

With the exception of convex weighting functions, explored by several authors, the parametric weighting functions proposed in the literature are generally consistent with bounded subadditivity. Setting $\varepsilon=.1$ and $\varepsilon^{\prime}=0$ is sufficient to accommodate most of these functions.

There are two approaches for testing SA, axiomatic and parametric. Wakker, Erev, and Weber (1994) found that in its general form the comonotonic independence axiom, which underlies all rank-dependent models, did not fare better than the independence axiom of expected utility, but "CPT with its $S$-shaped $w$-function provides the best description of the choice patterns ob-

\footnotetext{
${ }^{9}$ The same boundary constant $\varepsilon$ applies to (4.1) and (4.4), and the same boundary constant $\varepsilon^{\prime}$ to (4.2) and (4.3).
} 
served in this experiment. However, the improvement in prediction does not reach statistical significance" (p. 214). Stronger support for the preference conditions for an $S$-shaped weighting function was obtained by $\mathrm{Wu}$ and Gonzales (1994).

Most studies have estimated decision weights on the basis of various parametric assumptions about the value or the weighting function. The resulting weighting functions generally supported SA (Hogarth and Einhorn (1990), Birnbaum, Coffey, Mellers, and Weiss (1992), Lattimore, Baker, and Witte (1992), Tversky and Kahneman (1992), Gonzales (1993), Camerer and Ho (1994), Tversky and Fox (1994)); for a recent review that includes some earlier literature, see Camerer (1994).

Violations of SA are rare. One of the four subjects in Allais (1988) exhibits a convex ("pessimistic") weighting function, which violates lower SA. In Lattimore, Baker, and Witte (1992), only five out of 114 subjects yielded estimates that were inconsistent with SA. Karmarkar (1978) and Karni and Safra (1990) also considered $S$-shaped weighting functions.

It is noteworthy that lower SA accounts for the observed tendency (Kahneman and Tversky (1979)) to undervalue probabilistic insurance that reduces the probability of a loss, say from $p$ to $p / 2$, relative to regular insurance that reduces it from $p$ to 0 .

In order to characterize the degree of departure from expected utility theory, it is useful to devise a measure of the degree of SA. To this end, define for given $p, q$ satisfying $p+q \leq 1$ and the appropriate boundary conditions,

$$
\begin{aligned}
& D(p, q)=w(p)+w(q)-w(p+q) \text { and } \\
& D^{\prime}(p, q)=1-w(1-q)+w(p)-w(p+q) .
\end{aligned}
$$

Under SA, both $D$ and $D^{\prime}$ are positive for all $p, q$ that satisfy the boundary conditions, whereas under expected utility $D$ and $D^{\prime}$ are both zero. Let $d$ and $d^{\prime}$ denote, respectively, the average values of $D$ over all $p+q \leq 1-\varepsilon$ and of $D^{\prime}$ over all $p \geq \varepsilon^{\prime}$.

Simple graphical interpretations are possible whenever the weighting function is approximately linear except near the endpoints; see Figure 3. In this case, for all $p, q$ in the linear middle range, $D$ and $D^{\prime}$ are independent of $p$ and $q$, and $D$ is the lower intercept, $D^{\prime}$ the upper intercept, of the linear function. Thus the averages $d$ and $d^{\prime}$ provide estimates for the lower and upper intercepts, and $s=1-d-d^{\prime}$ is an estimate of the slope; $s$ can be interpreted as an index of sensitivity to probability changes. It equals 1 for expected utility, and it is less than 1 under SA. If expected utility is accepted as a standard for rational choice, then $s$ could be interpreted as an index of rationality.

Tversky and Fox (1994) estimated the values of $d, d^{\prime}$, and $s$ for three studies of risky choice. The median estimates were $.07, .16$, and .76 , in accord with both lower and upper SA. The observation that $d^{\prime}$ exceeds $d$ suggests that upper SA is generally more pronounced than lower SA. Further discussion of the empirical evidence appears in the final section. 


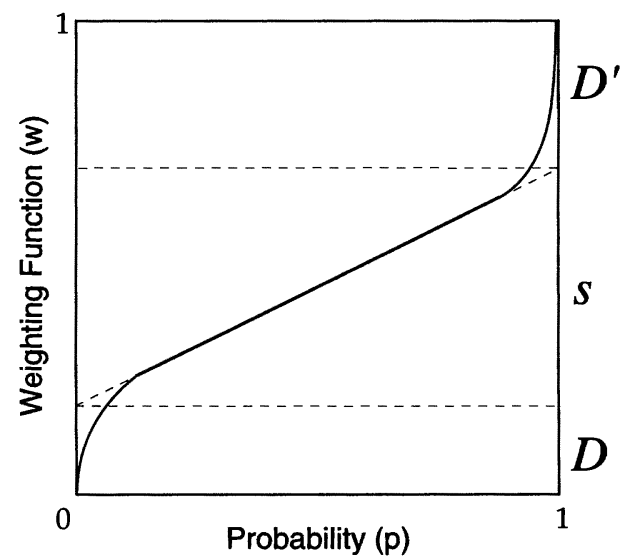

Figure 3.-A weighting function that is linear except near the endpoints.

\section{SUBADDITIVITY IN CHOICE UNDER UNCERTAINTY}

The above definitions of SA naturally extend to uncertainty: $W$ satisfies bounded subadditivity, or subadditivity $(S A)$ for short, ${ }^{10}$ if there are events $E, E^{\prime}$ such that

$$
W(B) \geq W(A \cup B)-W(A) \text { whenever } \quad W(A \cup B) \leq W(S-E)
$$

and

$$
1-W(S-B) \geq W(A \cup B)-W(A) \text { whenever } W(A) \geq W\left(E^{\prime}\right) .
$$

Conditions (5.1) and (5.2) are called lower $S A$ and upper $S A$, respectively. Lower SA implies that an event $B$ has a greater impact when added to the null event than when it is added to a nonnull event $A$. Upper SA implies that an event $B$ has a greater impact if it is subtracted from certainty than when it is subtracted from an event $A \cup B$. Upper SA for $W$ is equivalent to lower SA for the dual weighting function $\hat{W}(A)=1-W(S-A)$. The events $E, E^{\prime}$ are called lower and upper boundary events. They are "small" events that do not depend on $A$ and $B$. Under SA, an event $B$ has greater impact when it turns impossibility into possibility or possibility into certainty than when it merely makes a possibility more likely. That is, a change from $\varnothing$ to $B$, or from $S-B$ to $S$, is more noticeable than a change from $A$ to $A \cup B$.

\section{Preference Conditions}

The preference conditions for uncertainty are similar to those for risk, but require one further preparation; because the probabilities are not given, inequalities such as $W(A) \geq W\left(E^{\prime}\right)$ must be defined in terms of preferences. This is commonly done by defining $A \geqslant B$ if there exists a gain $Z$ such that

\footnotetext{
${ }^{10}$ Again, we use the terms bounded SA or SA instead of the more accurate $E, E^{\prime}$-SA.
} 
TABLE II

A Demonstration of SA. Outcomes Depend on the Temperature $t$ at 4 PM ON April 1, 1991 in New York City. The Percentage of Respondents

(113 Stock Brokers) who Selected Each Prospect is Given in Brackets, SEPARATELY FOR GAINS $(K=\$ 1000)$ AND FOR LOSSES $(K=-\$ 1000)$.

\begin{tabular}{clccccccc}
\hline Problem & & $\begin{array}{c}A_{1} \\
\text { if } t>80\end{array}$ & $\begin{array}{c}A_{2} \\
\text { if } 80 \geq t \geq 70\end{array}$ & $\begin{array}{c}A_{3} \\
\text { if } 70>t \geq 60\end{array}$ & $\begin{array}{c}A_{4} \\
\text { if } 60>t\end{array}$ & Gains & Losses \\
\hline \multirow{2}{*}{ I } & $f^{\prime}$ & 0 & 0 & 0 & $5 K$ & {$[65]$} & {$[34]$} \\
& $g^{\prime}$ & $2 K$ & $2 K$ & 0 & 0 & {$[35]$} & {$[66]$} \\
\multirow{2}{*}{ II } & $f^{\prime \prime}$ & $5 K$ & 0 & 0 & 0 & {$[58]$} & {$[29]$} \\
& $g^{\prime \prime}$ & 0 & 0 & $2 K$ & $2 K$ & {$[42]$} & {$[71]$} \\
\multirow{2}{*}{ III } & $f$ & $5 K$ & 0 & 0 & $5 K$ & {$[32]$} & {$[72]$} \\
& $f$ & $2 K$ & $2 K$ & $2 K$ & $2 K$ & {$[68]$} & {$[28]$} \\
\hline
\end{tabular}

$(Z, A) \geqslant(Z, B)$, that is, winning on $A$ is preferred to winning on $B .{ }^{11}$ Clearly, $A \geqslant B$ if and only if $W(A) \geq W(B)$. The following two conditions characterize SA:

$$
\begin{aligned}
z & \sim(0, S-(A \cup B) ; z, B ; Z, A) \quad \text { implies } \\
(z, S-B ; Z, B) & \geqslant(Z, A \cup B)
\end{aligned}
$$

whenever $0<z<Z$ and $A \cup B \preccurlyeq S-E$, and

$$
\begin{aligned}
(z, S-B) & \sim(Z, A) \quad \text { implies } \\
z & \geqslant(0, S-(A \cup B) ; z, B ; Z, A),
\end{aligned}
$$

whenever $0<z<Z$ and $A \geqslant E^{\prime}$; here $E$ and $E^{\prime}$ are the boundary events. The interpretation of (5.3) and (5.4) is similar to that of the corresponding conditions for risk. To see that lower SA implies (5.3), note that the CPT difference between the left prospects in $(5.3)$ is $W(B)(v(Z)-v(z))$, and between the right prospects is $(W(A \cup B)-W(A))(v(Z)-v(z))$. To derive (5.4) from upper SA, note that the CPT difference between the left prospects in (5.4) is (1$W(S-B)) v(z)$, and between the right prospects it is $(W(A \cup B)-W(A)) v(z)$.

Proposition 5.1: Under Assumption 3.1, the weighting function $W$ satisfies $S A$ if and only if (5.3) and (5.4) are satisfied. ${ }^{12}$

We conclude the section with several empirical observations. Table II illustrates both upper and lower SA, and provides a novel counterexample to expected utility that does not involve independence or substitution.

Table II shows that for the gain prospects the majority choice favored $f^{\prime}$ over $g^{\prime}, f^{\prime \prime}$ over $g^{\prime \prime}$, and $g$ over $f$, although $f=f^{\prime}+f^{\prime \prime}$ and $g=g^{\prime}+g^{\prime \prime}$. Furthermore,

${ }^{11}$ For losses a dual definition should be used. That is, $A$ is more likely than $B$ if there exists a loss $-Z$ such that $(-Z, A) \preccurlyeq(-Z, B)$.

${ }^{12}$ The same boundary event $E$ applies to (5.1) and (5.3), and the same boundary event $E^{\prime}$ applies to (5.2) and (5.4). 
$\left(f^{\prime}, f^{\prime \prime}, g\right)$ was the single most popular pattern for gains, exhibited by $31 \%$ of the subjects. This pattern violates expected utility, but is consistent with SA. To verify this, note that lower SA implies $W\left(A_{1} \cup A_{4}\right) \leq W\left(A_{1}\right)+W\left(A_{4}\right)$. Furthermore, if upper SA exceeds lower SA (i.e., $E^{\prime}=\varnothing$ can be taken), as is commonly the case, then $1 \geq W\left(A_{1} \cup A_{2}\right)+W\left(A_{3} \cup A_{4}\right)$ follows. Therefore, $V(f) \leq V\left(f^{\prime}\right)$ $+V\left(f^{\prime \prime}\right)$ but $V(g) \geq V\left(g^{\prime}\right)+V\left(g^{\prime \prime}\right)$, where $V(f)$ denotes the value of prospect $f$ according to CPT. Hence the observed pattern is consistent with CPT. Because the data exhibit SA in a strict sense, they are inconsistent with expected utility theory, in which (setting the utility of 0 to 0 ) all the above inequalities should be equalities.

Note that the modal preferences for the loss prospects are the mirror image of the preferences for the gain prospects, again exhibiting SA in the strict sense contrary to expected utility. Here, $\left(g^{\prime}, g^{\prime \prime}, f\right)$ was the single most popular pattern, exhibited by $35 \%$ of the subjects. This pattern is consistent with the reflection assumption $\left(W^{+}=W^{-}\right)$, characterized in Appendix B. ${ }^{13}$

Although SA is a plausible condition for decision under uncertainty, it is unlikely to hold in some special circumstances in which the union of disjoint events is less "vague" than its constituents. For example, consider an urn with one hundred green and red balls in unknown proportion, which are numbered from 1 to 100 . Then the events "even and red" and "even and green" are vague, but their union "even" is no longer vague. If, as suggested by Ellsberg (1961), people prefer to bet on known probabilities, defined by the numbers, rather than on the unknown probabilities involving colors, then SA may not hold in such situations.

\section{COMPARATIVE SUBADDITIVITY}

The relation more-concave-than or more-risk-averse-than between utility functions of different individuals was introduced and characterized by Pratt (1964) and Arrow (1965). This relation orders individuals by their departure from the (objective) expected value. In this section we develop a similar analysis for weighting functions. Specifically, we introduce and characterize the relation of more-SA-than between the weighting functions of different individuals, which orders them by their departure from expected utility theory. If this theory is taken as the standard of rational behavior, then the more-SA-than relation can be interpreted as an ordering by departure from rationality.

As in Section 4, the present treatment extends previous work (Yaari (1987), Chew, Karni, and Safra (1989), Chew (1989), Chateauneuf (1991), Chateauneuf and Cohen (1994), Wakker (1994)) by considering $S$-shaped rather than convex weighting functions, and by comparing weighting functions independently of the value functions.

\footnotetext{
${ }^{13}$ The modal choices in Table II are also at variance with additive regret models (Bell (1982), Loomes and Sugden (1982), and Fishburn (1982)), which are violated by the strict form of (5.3) and (5.4).
} 
A transformation $\phi:[0,1] \rightarrow[0,1]$ is called $S A$ if it has the same mathematical properties as an SA weighting function, i.e., $\phi(0)=0, \phi(1)=1, \phi$ is continuous and strictly increasing, and $\phi$ satisfies (4.1) and (4.2). One weighting function is more $S A$ than another, if the first is obtained from the second by an SA transformation. This definition applies to both risk and uncertainty, where the weighting functions for uncertainty are defined on the same domain. Figure 4 illustrates this relation.

\section{Decision under Risk}

It is readily verified (see Proposition 6.1) that $w_{2}$ is more SA than $w_{1}$ if and only if the following two conditions hold:

$$
\begin{aligned}
& w_{1}(r)=w_{1}(p+q)-w_{1}(p) \quad \text { implies } \\
& w_{2}(r) \geq w_{2}(p+q)-w_{2}(p)
\end{aligned}
$$

and

$$
\begin{aligned}
& 1-w_{1}(1-r)=w_{1}(p+q)-w_{1}(p) \quad \text { implies } \\
& 1-w_{2}(1-r) \geq w_{2}(p+q)-w_{2}(p) ;
\end{aligned}
$$

the boundary condition for (6.1) is $p+q \leq 1-\varepsilon$ for $\varepsilon \geq 0$, and the boundary

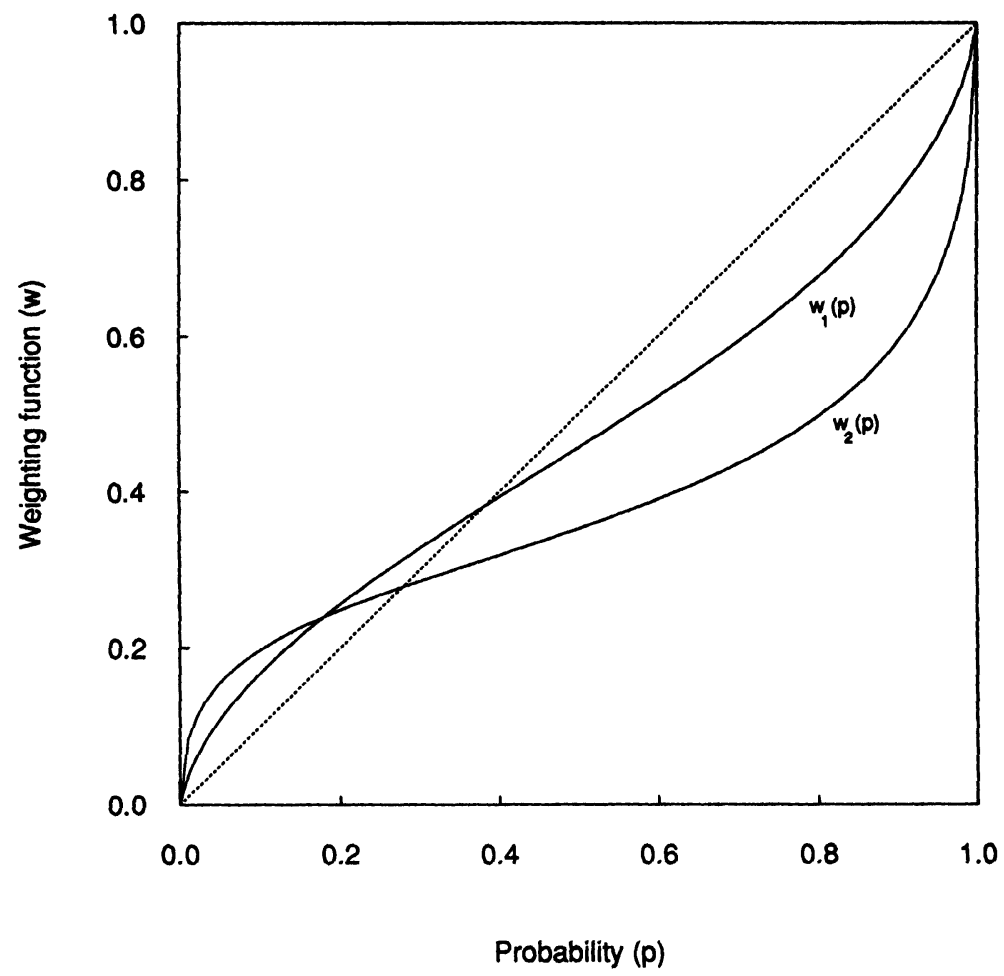

FIGURE 4. - The weighting function $w_{2}$ is less additive than $w_{1}$. 
condition for (6.2) is $p \geq \varepsilon^{\prime}$, where $\varepsilon^{\prime} \geq 0$. The proof is based on the observation that, under (6.1), $w_{2}$ satisfies lower SA "when its arguments are measured in $w_{1}$ units." The same logic applies to (6.2). The relation of $\varepsilon$ and $\varepsilon^{\prime}$ to the boundary constants for the transformation carrying $w_{1}$ into $w_{2}$ is given in Appendix A; see (A1) and (A2). According to the above conditions, $w_{2}(r)$ and $\hat{w}_{2}(r)=1-w_{2}(1-r)$ are overweighted more than $w_{1}(r)$ and $\hat{w}_{1}(r)=1-$ $w_{1}(1-r)$, respectively.

Next we present the preference conditions for the proposition that $\succcurlyeq_{2}$ is more SA than $\succcurlyeq_{1}$, defined for the preference orders of two individuals. We impose no restrictions on the value functions of the two individuals, so their value functions may be different. Two conditions are required, one for upper and one for lower SA. First we consider lower SA:

$$
\begin{aligned}
\text { If } x & \sim_{1}(0,1-p-q ; x, q ; X, p) \text { and } \\
(x, 1-r ; X, r) & \sim_{1}(X, p+q), \text { then } \\
y & \sim_{2}(0,1-p-q ; y, q ; Y, p) \text { implies } \\
(y, 1-r ; Y, r) & \succcurlyeq_{2}(Y, p+q),
\end{aligned}
$$

whenever $0<x<X, 0<y<Y$, and $p+q \leq 1-\varepsilon$ for the boundary constant $\varepsilon \geq 0$. This condition states that if, for $\succcurlyeq_{1}$, the improvement on the left ( $r$ probability for receiving $X$ instead of $x$ ) matches the corresponding improvement on the right, then for $\succcurlyeq_{2}$ the comparable improvement on the left ( $r$ probability for receiving $Y$ instead of $y$ ) outweighs the corresponding improvement on the right. To further illustrate the condition, we show how it follows from (6.1). The first two indifferences imply, by comparing the CPT difference of the left prospects to that of the right prospects, that $w_{1}(r)\left(v_{1}(X)-v_{1}(x)\right)=$ $\left(w_{1}(p+q)-w_{1}(p)\right)\left(v_{1}(X)-v_{1}(x)\right)$, i.e., $w_{1}(r)=w_{1}(p+q)-w_{1}(p)$. By $(6.1), w_{2}(r)$ $\geq w_{2}(p+q)-w_{2}(p)$. This implies that $w_{2}(r)\left(v_{2}(Y)-v_{2}(y)\right)$, i.e., the CPT difference between the left prospects in the lower two lines in (6.3), is at least as large as $\left(w_{2}(p+q)-w_{2}(p)\right)\left(v_{2}(Y)-v_{2}(y)\right)$, which is the difference between the right two prospects. Hence (6.1) implies (6.3).

Second, we consider upper SA:

$$
\text { If } \begin{aligned}
(x, 1-r) & \sim_{1}(X, p) \text { and } \\
x & \sim_{1}(0,1-p-q ; x, q ; X, p) \text { then } \\
(y, 1-r) & \sim_{2}(Y, p) \text { implies } \\
y & \succcurlyeq_{2}(0,1-p-q ; y, q ; Y, p),
\end{aligned}
$$

whenever $0<x<X, 0<y<Y$, and $p \geq \varepsilon^{\prime}$ for the boundary constant $\varepsilon^{\prime} \geq 0$. This condition states that if, for $\succcurlyeq_{1}$, the improvement on the left (yielding $x$ with certainty) matches the corresponding improvement on the right, then for $\succcurlyeq_{2}$ the comparable improvement on the left (yielding $y$ with certainty) outweighs the corresponding improvement on the right. In other words, the certainty effect is more pronounced for the second decision maker than for the first. 
PROPOSITION 6.1: Under Assumption 3.1 ( for both $\succcurlyeq_{1}$ and $\succcurlyeq_{2}$ ), the following three statements are equivalent: ${ }^{14}$

(i) $w_{2}$ is more $S A$ than $w_{1}$;

(ii) conditions (6.1) and (6.2) are satisfied;

(iii) conditions (6.3) and (6.4) are satisfied.

\section{Decision under Uncertainty}

We first extend (6.1) and (6.2) to uncertainty. $W_{2}$ is more SA than $W_{1}$ if and only if (see Proposition 6.2) $W_{2}$ is a strictly increasing transform of $W_{1}$, and

$$
\begin{aligned}
& W_{1}(C)=W_{1}(A \cup B)-W_{1}(A) \quad \text { implies } \\
& W_{2}(C) \geq W_{2}(A \cup B)-W_{2}(A)
\end{aligned}
$$

and

$$
\begin{aligned}
& 1-W_{1}(S-C)=W_{1}(A \cup B)-W_{1}(A) \quad \text { implies } \\
& 1-W_{2}(S-C) \geq W_{2}(A \cup B)-W_{2}(A) .
\end{aligned}
$$

The boundary condition for (6.5) is: $W_{1}(A \cup B) \leq W_{1}(S-E)$ for some boundary event $E$; the boundary condition for (6.6) is: $W_{1}(A) \geq W_{1}\left(E^{\prime}\right)$ for a boundary event $E^{\prime}$. The relation of $E$ and $E^{\prime}$ to the boundary constants for the transformation carrying $W_{1}$ into $W_{2}$ is given in (A3) and (A4) in the Appendix.

Next we present the corresponding preference conditions for the proposition that $\succcurlyeq_{2}$ is more SA than $\succcurlyeq_{1}$. We require three conditions.

First, for all events $A, B$,

$$
A \succcurlyeq_{1} B \text { if and only if } A \succcurlyeq_{2} B \text {. }
$$

This condition guarantees that $W_{2}$ is a strictly increasing transform of $W_{1}$. The second condition ensures that $W_{2}$ is more lower SA than $W_{1}$ :

$$
\begin{aligned}
\text { If } x & \sim_{1}(0, S-(A \cup B) ; x, B ; X, A) \text { and } \\
(x, S-C ; X, C) & \sim_{1}(X, A \cup B) \text { then } \\
y & \sim_{2}(0, S-(A \cup B) ; y, B ; Y, A) \text { implies } \\
(y, S-C ; Y, C) & \succcurlyeq_{2}(Y, A \cup B)
\end{aligned}
$$

whenever $0<x<X, 0<y<Y$, and $A \cup B \preccurlyeq_{1} S-E$ for the boundary event $E$. Third, we require that $W_{2}$ be more upper SA than $W_{1}$ :

$$
\text { If } \begin{aligned}
(x, S-C) & \sim_{1}(X, A) \text { and } \\
x & \sim_{1}(0, S-(A \cup B) ; x, B ; X, A) \text { then } \\
(y, S-C) & \sim_{2}(Y, A) \text { implies } \\
y & \succcurlyeq_{2}(0, S-(A \cup B) ; y, B ; Y, A)
\end{aligned}
$$

\footnotetext{
${ }^{14}$ The same boundary constants $\varepsilon, \varepsilon^{\prime}$ apply to conditions (6.1) and (6.2), and to conditions (6.3) and (6.4). Their relation to the boundary constants for the transformation carrying $w_{1}$ into $w_{2}$ is described in (A1) and (A2) in the Appendix.
} 
whenever $0<x<X, 0<y<Y$, and $A \succcurlyeq_{1} E^{\prime}$ for the boundary event $E^{\prime}$. The interpretation of (6.8) and (6.9) is essentially identical to that of (6.3) and (6.4).

Proposition 6.2: Under Assumption 3.1 ( for both $\succcurlyeq_{1}$ and $\succcurlyeq_{2}$ ), the following three statements are equivalent: $:^{15}$

(i) $W_{2}$ is more $S A$ than $W_{1}$;

(ii) conditions (6.5), (6.6), and (6.7) are satisfied;

(iii) conditions (6.7), (6.8), and (6.9) are satisfied.

\section{SOURCE DEPENDENCE}

Perhaps the most persistent objection to expected utility theory concerns the distinction between risk and uncertainty. The expectation principle, it has been argued, can be applied to decision under risk where probabilities are known but not to decision under uncertainty or ignorance where the probabilities are unknown. This view, advanced by several authors, notably Keynes (1921) and Knight (1921), has been underscored by Ellsberg (1961), who argued convincingly that people prefer to bet on an urn that contains an equal number of green and red balls than on an urn that contains red and green balls in an unknown proportion. Numerous experiments have confirmed this hypothesis; see Camerer and Weber (1992) for a review. More generally, there is evidence that people's preferences depend not only on their degree of uncertainty but also on the source of uncertainty. This phenomenon has been called source dependence.

In this section we distinguish two aspects of source dependence, which we call source preference and source sensitivity. Let $\mathscr{A}$ and $\mathscr{B}$ be two distinct families of events. For example, one family may be generated by spinning a roulette wheel, the other by the possible outcomes of a horse race. We shall refer to such families as sources. We assume that the families are closed under union and complementation, and are rich in the sense that they both satisfy solvability. In decision under risk, we interpret the uncertainty as generated by a standard random device. Although probabilities could be realized by various random devices, we do not distinguish between them and treat risk as a single source.

\section{Source Preference}

In the domain of gains, the decision maker exhibits a general preference for source $\mathscr{A}$ over source $\mathscr{B}$ if, for any event $A$ in $\mathscr{A}$ and $B$ in $\mathscr{B}, W^{+}(A)=W^{+}(B)$ implies $W^{+}(S-A) \geq W^{+}(S-B)$. Expressed in terms of preferences, this means that $(x, A) \sim(x, B)$ implies $(x, S-A) \geqslant(x, S-B)$ for all $x>0$. Ellsberg's example of preference for the known over the unknown urn illustrates this relation.

To extend source preference to negative outcomes, we start with the preference condition. A preference to bet on source $\mathscr{A}$ rather than $\mathscr{B}$ means that

\footnotetext{
${ }^{15}$ The same boundary events $E, E^{\prime}$ apply to conditions (6.5) and (6.6), and to conditions (6.8) and (6.9). Their relation to the boundary constants for the transformation carrying $W_{1}$ into $W_{2}$ is described in (A3) and (A4) in the Appendix.
} 
$(-x, A) \sim(-x, B)$ implies $(-x, S-A) \succcurlyeq(-x, S-B)$ for any $x>0$. In terms of the weighting function, therefore, source $\mathscr{A}$ is preferred to source $\mathscr{B}$ if $W^{-}(A)$ $=W^{-}(B)$ implies $W^{-}(S-A) \leq W^{-}(S-B)$. Note that the inequality for losses is the opposite of the inequality for gains. Hence source preference reduces the weighting function for losses and enhances the weighting function for gains. Indeed, it can be shown that if reflection holds, then source $\mathscr{A}$ is preferred to source $\mathscr{B}$ for gains if and only if $\mathscr{B}$ is preferred to $\mathscr{A}$ for losses. Consequently, reflection cannot be satisfied if $\mathscr{A}$ is preferred to $\mathscr{B}$ for both gains and losses.

\section{Source Sensitivity}

We next examine the concept of source sensitivity (SS). In the domain of gains, the decision maker exhibits less $S S$ to source $\mathscr{B}$ than to source $\mathscr{A}$ if the following two conditions hold:

$$
\begin{aligned}
& \text { if } W^{+}\left(A_{1}\right)=W^{+}\left(B_{1}\right) \text { and } W^{+}\left(A_{2}\right)=W^{+}\left(B_{2}\right), \\
& \text { then } W^{+}\left(A_{1} \cup A_{2}\right) \geq W^{+}\left(B_{1} \cup B_{2}\right) \text {; } \\
& \text { if } W^{+}\left(S-A_{1}\right)=W^{+}\left(S-B_{1}\right) \text { and } W^{+}\left(S-A_{2}\right)=W^{+}\left(S-B_{2}\right) \text {, } \\
& \text { then } W^{+}\left(S-\left(A_{1} \cup A_{2}\right)\right) \leq W^{+}\left(S-\left(B_{1} \cup B_{2}\right)\right)
\end{aligned}
$$

for all disjoint events $A_{1}, A_{2}$ in $\mathscr{A}$ and disjoint events $B_{1}, B_{2}$ in $\mathscr{B}$ satisfying the following boundary conditions. In (7.1), $W^{+}\left(A_{1} \cup A_{2}\right) \leq W^{+}(S-E)$ for some boundary event $E$; in (7.2), $W^{+}\left(S-\left(A_{1} \cup A_{2}\right)\right) \geq W^{+}\left(E^{\prime}\right)$ for some boundary event $E^{\prime}$. Conditions (7.1) and (7.2) are dual in the sense that one condition holds if and only if the other holds for the dual weighting function.

To appreciate the above definition, suppose $W^{+}$is SA on $\mathscr{A}$ and on $\mathscr{B}$. Equation (7.1) means that the union of disjoint $\mathscr{B}$ events "loses" more than the union of the matching disjoint $\mathscr{A}$ events. Hence the decision maker is less sensitive to an increase in likelihood in source $\mathscr{B}$ than in source $\mathscr{A}$. Equation (7.2) imposes the dual condition. The comparative SS relation between sources for the same decision maker is reminiscent of the more-SA-than relation between different decision makers for the same source. Both relations reflect departure from expected utility, but they are formally and conceptually different.

Expressed in terms of preferences, (7.1) is equivalent to:

$$
\begin{aligned}
\left(x, A_{1}\right) & \sim\left(x, B_{1}\right) \text { and }\left(x, A_{2}\right) \sim\left(x, B_{2}\right) \text { implies } \\
\left(x, A_{1} \cup A_{2}\right) & \geqslant\left(x, B_{1} \cup B_{2}\right) \text { for any } x>0,
\end{aligned}
$$

and (7.2) is equivalent to:

$$
\begin{aligned}
\left(x, S-A_{1}\right) & \sim\left(x, S-B_{1}\right) \text { and }\left(x, S-A_{2}\right) \sim\left(x, S-B_{2}\right) \text { implies } \\
\left(x, S-\left(A_{1} \cup A_{2}\right)\right) & \preccurlyeq\left(x, S-\left(B_{1} \cup B_{2}\right)\right) \text { for any } x>0,
\end{aligned}
$$

for disjoint $A_{1}, A_{2}$ and disjoint $B_{1}, B_{2}$, and under the boundary conditions for (7.1) and (7.2), respectively. The relations of source preference and comparative SS are logically independent. If people prefer one source to another, they can exhibit more or less SS for one source than for the other, or neither. 
Turning to losses, we say that the decision maker exhibits less $S S$ to source $\mathscr{B}$ than to source $\mathscr{A}$ if conditions (7.1) and (7.2) hold for the weighting function for losses. The preference conditions are obtained from (7.3) and (7.4) by interchanging $x>0$ and $x<0$, as well as $\succcurlyeq$ and $\preccurlyeq$.

\section{Empirical Evidence}

We conclude this section by discussing some experimental demonstrations of SA and source preference. We define, for any disjoint events $A, B$ satisfying the appropriate boundary condition,

$$
\begin{aligned}
& D(A, B)=W(A)+W(B)-W(A \cup B) \text { and } \\
& D^{\prime}(A, B)=1-W(S-B)-W(A \cup B)+W(A) .
\end{aligned}
$$

Unlike the case of risk, there is no obvious way to define the global measures $d$ or $d^{\prime}$, because there is no natural prior measure on the event space with respect to which the averages could be taken. However, for any given experiment one can compute the average of the indices $d, d^{\prime}$ over all disjoint event pairs $A, B$. Again, $s=1-d-d^{\prime}$ is a measure of correspondence with expected utility.

Tversky and Fox (1994) estimated decision weights for six sources, including risk. The risky events were generated by drawing a ball from an urn with known composition. The other sources consisted of the following uncertain quantities: the point spread in a playoff basketball game, the point spread of the 1992 Superbowl, the difference between the closing values of the Dow Jones on successive weeks, San Francisco temperature, and Beijing temperature. Table III presents the median values of $d, d^{\prime}$, and $s$, for all sources of uncertainty in each of the three studies.

All values of $d$ and $d^{\prime}$ in Table III, which measure lower and upper SA respectively, are significantly greater than zero, confirming SA for all sources including chance. Furthermore, $d$ and $d^{\prime}$ are greater (and hence $s$ is smaller) for uncertainty than for chance, indicating that people are less sensitive to uncertainty than to chance.

Figure 5 displays, for each subject in one study $(N=40)$, the average $s$ value for the two uncertain sources (Super Bowl and Dow Jones) against the $s$ value for chance. Three features of Figure 5 are noteworthy. First, all values of $s$ for the uncertain sources, and all but two values of $s$ for the risky source are less

TABLE III

Median Values of $d$ and $d$, and $s$, Across Subjects, Measuring the Degree

\begin{tabular}{|c|c|c|c|c|c|c|c|c|c|}
\hline \multirow[b]{2}{*}{ Source } & \multicolumn{3}{|c|}{ Study 1} & \multicolumn{3}{|c|}{ Study 2} & \multicolumn{3}{|c|}{ Study 3} \\
\hline & $d$ & $d^{\prime}$ & $s$ & $d$ & $d^{\prime}$ & $s$ & $d$ & $d^{\prime}$ & $s$ \\
\hline Chance & .06 & .10 & .81 & .05 & .19 & .75 & .11 & .14 & .72 \\
\hline Basketball & .21 & .19 & .61 & & & & & & \\
\hline Super Bowl & & & & .15 & .23 & .57 & & & \\
\hline Dow Jones & & & & .12 & .22 & .67 & & & \\
\hline S.F. temp. & .20 & .26 & .51 & & & & .27 & .23 & .50 \\
\hline Beijing temp. & & & & & & & .28 & .32 & .42 \\
\hline
\end{tabular}
of LOWER SA, UPPER SA, AND GLOBAL SENSITIVITY RESPECTIVELY. 


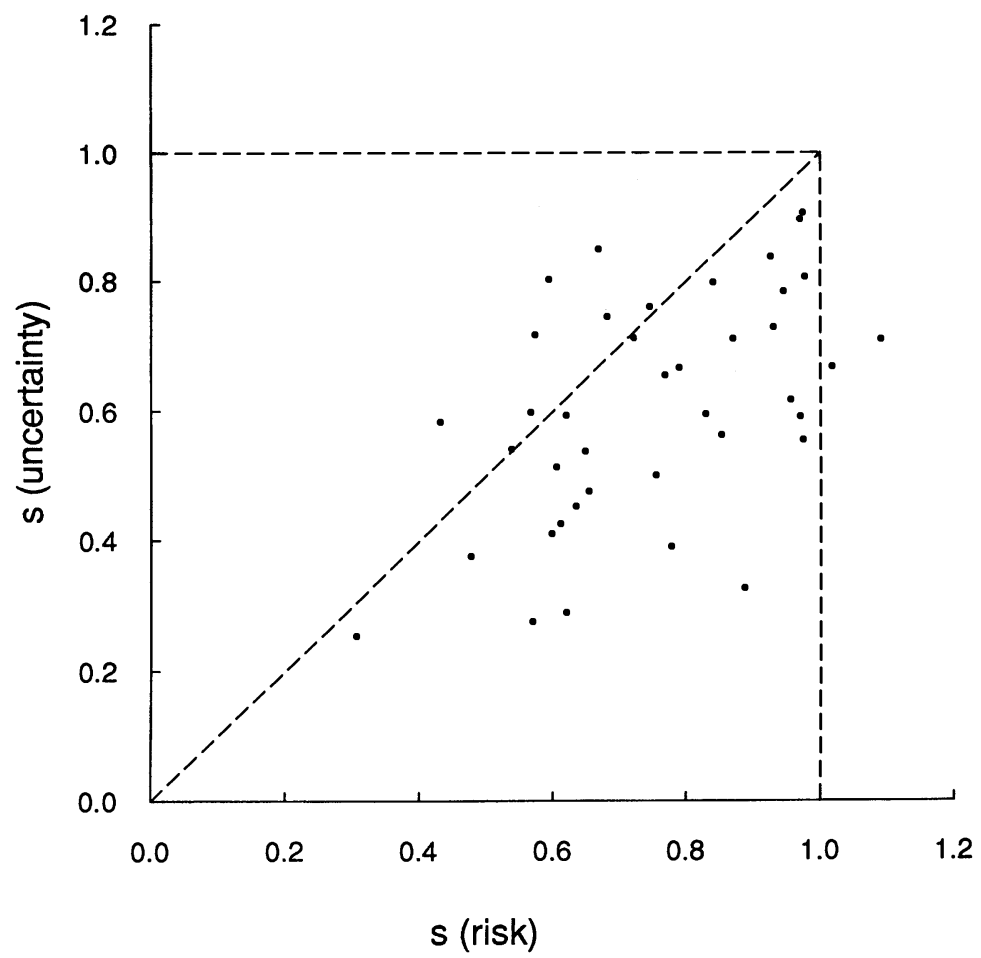

FIGURE 5.-Joint distribution for all subjects in study 2 of the sensitivity measure $s$ for risk and uncertainty.

than 1 , as implied by SA. Second, the sensitivity measure $s$ is significantly smaller for uncertainty (mean $=.61$ ) than for risk (mean $=.75)$; 32 out of 40 points lie below the identity line. Third, there is a significant correlation $(r=.53)$ between the sensitivity measure for risk and for uncertainty, suggesting that the degree of SA (as measured by $s$ ) is an important attribute that distinguishes among decision makers.

In addition to the indirect comparisons in terms of $s$ described above, Tversky and Fox also tested the preference conditions for comparative SS. The ordinal analysis confirmed the previous conclusion: subjects exhibited less SS for all five uncertain sources than for chance in the sense that (7.3) and (7.4) were satisfied significantly more often in the predicted than in the opposite direction.

Finally, the evidence indicated that some sources were preferred to risk. For example, Stanford students (who lived near San Francisco) preferred to bet on San Francisco temperature than on risk, but they preferred to bet on risk than on Beijing temperature.

In summary, it appears that the characteristics of the weighting function, which have been observed in studies of risk, tend to hold for uncertainty as well. Hence SA emerges as an important descriptive principle for decision under both risk and uncertainty. Furthermore, the finding that people are less sensitive to 
uncertainty than to risk indicates that uncertainty enhances the departures from expected utility. Studies of choice under risk therefore provide a lower bound for the departure from expected utility caused by nonadditive weights. Finally, the observation that people often prefer to bet on unknown rather than known probabilities calls for a reassessment of the conclusion commonly drawn from Ellsberg's example. It appears that people prefer risk to uncertainty when they are made to feel ignorant or incompetent. However, in other situations people often prefer betting on an uncertain source (e.g., sports or weather) than on risk (Heath and Tversky (1991)). A comprehensive analysis of the causes and consequences of source dependence awaits further theoretical and experimental research.

\section{Psychology Dept., Stanford University, Stanford, CA 94305, U.S.A. and}

Medical Decision Making Unit, Uñiversity of Leiden, P.O. Box 9600, 2300 RC Leiden, The Netherlands

Manuscript received November, 1993; final revision received January, 1995.

\section{APPENDIX A: PROOFS}

Proof of Proposition 4.1: That the preference conditions are implied by the corresponding properties for weighting functions, was already explained in the text. Next we assume that (4.4) holds and derive (4.1). Let $p, q$ be such that $p+q \leq 1-\varepsilon$.

Case 1: $p=0$. Then (4.1) is trivially satisfied.

Case 2: $p>0$ and $p+q<1$. Then, by continuity of $v$, outcomes $Z>z>0$ can always be found to give the antecedent in (4.4). For any such outcomes, the CPT difference between the left prospects in (4.4) is $w(q)(v(Z)-v(z))$, the CPT difference between the right prospects is $(w(p+q)-$ $w(p))(v(Z)-v(z))$. By positivity of $v(Z)-v(z)$, the consequent preference in (4.4) holds iff $w(q) \geq w(p+q)-w(p)$. Thus (4.1) follows.

Case 3: $p>0, p+q=1$. (Note that $p+q=1$ can only occur if $\varepsilon=0$.) Define $p_{j}=p-1 / j$ for $j$ so large that $p_{j}>0$. By Case $2, w(q) \geq w\left(p_{j}+q\right)-w\left(p_{j}\right)$ for all such $p_{j}$; obviously, $p_{j}+q \leq 1-\varepsilon$. By continuity of $w,(4.1)$ follows for $p$.

Before turning to the implication (4.3) $\Rightarrow(4.2)$, let us briefly comment on the duality between (4.3) and (4.4). It can be seen that (4.4), when formulated in a perfectly dual manner, would lead to the preference condition $z \sim(0,1-p-q ; z, q ; Z, p) \Rightarrow(z, 1-q) \preccurlyeq(Z, p)$ (under appropriate boundary condition). This condition could have been used instead of (4.3) to characterize upper SA of $w$. We think, however, that the slightly different condition in (4.3) (logically equivalent under Assumption 3.1) is more transparent. Therefore the proof below is not a complete dual to the proof of the implication $(4.4) \Rightarrow(4.1)$.

Let us now assume that (4.3) holds and derive (4.2). Let $p, q$ be such that $p \geq \varepsilon^{\prime}$.

Case 1: $p=1-q$. Then (4.2) is trivially satisfied.

Case 2: $0<p<1-q$. Here outcomes $Z>z>0$ can always be found to give the antecedent in (4.3). For any such outcomes, the CPT difference between the left prospects in (4.3) is $(1-w(1-$ $q)) v(z)$, the CPT difference between the right prospects is $(w(p+q)-w(p)) v(z)$. By positivity of $v(z)$, the consequent preference in (4.3) holds iff $1-w(1-q) \geq w(p+q)-w(p)$; (4.2) follows.

Case 3: $0=p<1-q$. (Note that $p=0$ can only occur if $\varepsilon^{\prime}=0$.) Define $p_{j}=1 / j$ for $j$ so large that $p_{j}<1-q$. By Case $2,1-w(1-q) \geq w\left(p_{j}+q\right)-w\left(p_{j}\right)$ for all such $p_{j}$. By continuity of $w,(4.2)$ follows for $p=0$.

Q.E.D. 
Proof of Proposition 5.1: That the preference conditions are implied by the corresponding properties for weighting functions, was already explained in the text. Next we assume that (5.3) holds and derive (5.1). Suppose that $W(A \cup B) \leq W(S-E)$, i.e., $A \cup B \preccurlyeq S-E$. We may assume that $A, B$ are disjoint (the general case follows by substituting $A-B$ for $A$ ).

Case 1: $W(A)>0$ and $W(A \cup B)<1$. Then outcomes $Z>z>0$ can always be found to give the antecedent in (5.3). For any such outcomes, the CPT difference betwen the left prospects in (5.3) is $W(B)(v(Z)-v(z))$, the CPT difference between the right prospects is $(W(A \cup B)-W(A))(v(Z)-$ $v(z)$ ). By positivity of $v(Z)-v(z)$, the consequent preference in (5.3) holds iff $W(B) \geq W(A \cup B)-$ $W(A)$. (5.1) follows.

Case 2: $W(A)=0$ and $W(A \cup B)=1$. (Note that $W(A \cup B)=1$ can only occur if $W(S-E)=1$.) In this case the antecedent in (5.3) trivially holds, and the consequent preference in (5.3) implies $W(B)=1$. This implies (5.1).

Case 3: $W(A)=0$ and $W(A \cup B)<1$. This case is derived from Case 1 by a limiting argument and solvability of $W$. If $W(A \cup B)=0$ then (5.1) is immediate; therefore we assume $W(A \cup B)>0$. By solvability there exist, for all $n$ sufficiently large, events $A_{n}$ such that $A \subset A_{n} \subset A \cup B$ and $W\left(A_{n}\right)=1 / n$; define $B_{n}=B-A_{n}$. By Case $1, W\left(B_{n}\right) \geq W\left(A_{n} \cup B_{n}\right)-W\left(A_{n}\right)$; note here that the boundary condition is satisfied for $A_{n} \cup B_{n}(=A \cup B)$. Since $W(B) \geq W\left(B_{n}\right)$, it follows that $W(B) \geq$ $W(A \cup B)-W\left(A_{n}\right)$. In the limit (5.1) follows.

Case 4: $W(A)>0$ and $W(A \cup B)=1$. If $W(A)=1$ then (5.1) trivially holds; assume therefore $W(A)<1$. By solvability, for all $n$ sufficiently large there exist $B_{n} \subset B$ such that $W(A)<W\left(A \cup B_{n}\right)$ $=1-1 / n$. By Case $1, W\left(B_{n}\right) \geq W\left(A \cup B_{n}\right)-W(A)$; note that the boundary condition is satisfied for $A \cup B_{n}$. Because $W(B) \geq W\left(B_{n}\right), W(B) \geq W\left(A \cup B_{n}\right)-W(A)$. In the limit, $W(B) \geq W(A \cup B)$ $-W(A)$ follows, i.e., (5.1) holds.

Finally, we assume that (5.4) holds and derive (5.2). Suppose that $W(A) \geq W\left(E^{\prime}\right)$, i.e., $A \geqslant E^{\prime}$. We may assume that $A, B$ are disjoint (the general case follows by substituting $B-A$ for $B$ ). Note for the cases below that, by monotonicity of weighting functions, always $W(S-B) \geq W(A)$.

Case 1: $W(S-B)=W(A)$. Then (5.2) trivially holds.

Case 2: $W(S-B)>W(A)>0$. Then outcomes $Z>z>0$ can always be found to give the antecedent in (5.4). For any such outcomes, the CPT difference between the left prospects in (5.4) is $(1-W(S-B)) v(z)$; the CPT difference between the right prospects is $(W(A \cup B)-W(A)) v(z)$. By positivity of $v(z)$, the consequent preference in (5.4) holds iff $1-W(S-B)) \geq W(A \cup B)-W(A)$. (5.2) follows.

Case 3: $W(S-B)>W(A)=0$. (Note that $W(A)=0$ can only occur if $W\left(E^{\prime}\right)=0$.) By solvability there exist, for all $n$ sufficiently large, events $A_{n}$ such that $A \subset A_{n} \subset S-B$ and $W\left(A_{n}\right)=1 / n$. Note that $W\left(A_{n}\right) \geq W\left(E^{\prime}\right)=0$. By Case 2, $1-W(S-B) \geq W\left(A_{n} \cup B\right)-W\left(A_{n}\right)$. Because $A \subset A_{n}, 1-$ $W(S-B) \geq W(A \cup B)-W\left(A_{n}\right)$. In the limit $1-W(S-B) \geq W(A \cup B)-W(A)$ follows, i.e., (5.2) holds.

Q.E.D.

Proof of Proposition 6.1: We show how this result can be derived from the similar result for uncertainty, i.e, Proposition 6.2 (the proof of which is given below). Our proof thus illustrates the close relation between risk and uncertainty, and does not take much space. The disadvantage is, of course, that this proof of Proposition 6.1 is not self-contained but relies on Proposition 6.2.

Proposition 6.1 follows from Proposition 6.2 by setting $S=[0,1], P$ is the Lebesgue measure, $W_{1}=w_{1} \circ P$, and $W_{2}=w_{2} \circ P$. The only aspect that needs further discussion concerns the boundary constants. It was pointed out in the text that $w_{2}$ being more SA than $w_{1}$ can be viewed as $w_{2}$ satisfying SA "when its arguments are measured in $w_{1}$ units." Similarly, the boundary constraints for the SA transformation $\phi$ such that $w_{2}=\phi \circ w_{1}$ are simply the original boundary constraints, "measured in $w_{1}$ units." For an elaboration, denote by $\bar{\varepsilon}$ the boundary constant for condition (4.1), and by $\bar{\varepsilon}^{\prime}$ the one for (4.2), for transformation $\phi$. Then $p \geq \varepsilon^{\prime}$, the boundary condition for (6.2) and 
(6.4), corresponds with $w_{1}(p) \geq w_{1}\left(\varepsilon^{\prime}\right)\left(=W_{1}\left(E^{\prime}\right)\right.$ ) ("the values of $w_{1}$ serve as arguments of $\phi^{\prime \prime}$ ), and we get:

(A1) $\quad \bar{\varepsilon}^{\prime}=w_{1}\left(\varepsilon^{\prime}\right)$.

The boundary constraint for (6.1) and (6.3), i.e., $p+q \leq 1-\varepsilon$, corresponds to $w_{1}(p+q) \leq w_{1}(1-\varepsilon)$ $\left(=W_{1}(S-E)\right)$; therefore

(A2) $\quad 1-\bar{\varepsilon}=w_{1}(1-\varepsilon)$.

Q.E.D.

Proof of Proposition 6.2: In this proof we use the notation $\pi(B, A)$ for $W(A \cup B)-W(A)$, where it is implicitly assumed that $A$ and $B$ are disjoint; $\pi_{1}(B, A)$ and $\pi_{2}(B, A)$ are similar. Condition (6.7) is equivalent to $W_{2}=\phi \circ W_{1}$ for a strictly increasing transformation $\phi$, so that will be assumed from now on, and (6.7) is no more discussed. Note also that, because of solvability of both $W_{1}$ and $W_{2}$, the transformation $\phi$ is surjective, hence it must be continuous. Conditions (6.5) and (6.6) can be restricted to disjoint $A, B$, by replacing $B$ by $B-A$; this will be assumed below without further mention.

To derive equivalence of (i) and (ii) in the proposition, we first show that condition (6.5) is equivalent to condition (4.1) for the transformation $\phi$-the other conditions for $\phi, \phi(0)=0$, $\phi(1)=1$, and continuity and strict increașingness, have already been established. Condition (4.1) means that $\phi(b) \geq \phi(a+b)-\phi(a)$ ("the value of the difference is at least as large as the difference of values") whenever $a+b \leq 1-\bar{\varepsilon}$; here $\bar{\varepsilon}$ denotes the boundary constant for $\phi$, and is related to the boundary event $E$ in (6.5) by the equation

$$
1-\bar{\varepsilon}=W_{1}(S-E)
$$

as we shall see.

The proof is by substituting, along with (A3), $a+b=W_{1}\left(A \cup B\right.$ ) (for $A \cap B=\varnothing$ ), $a=W_{1}(A)$, $b=W_{1}(C)$; by continuity of $\phi$ and solvability of $W_{1}$, these substitutions can always be realized. The equality $W_{1}(C)=\pi_{1}(B, A)$ corresponds with $b=a+b-a$, the inequality $W_{1}(A \cup B) \leq W_{1}(S-E)$ corresponds with $a+b \leq 1-\bar{\varepsilon}$, and $W_{2}(C) \geq \pi_{2}(B, A)$ corresponds with $\phi(b) \geq \phi(a+b)-\phi(a)$. These substitutions show that (4.1) for $\phi$ is equivalent to (6.5) for $W_{1}, W_{2}$. It is demonstrated in a dual manner that (6.6) for $W_{1}, W_{2}$ is equivalent to (4.2) for $\phi$ (now $W_{1}(S-C)=1-b$ ). The relation between the boundary event $E^{\prime}$ and the boundary constant $\bar{\varepsilon}^{\prime}$ for the upper SA transformation $\phi$ is: ${ }^{16}$

$$
\bar{\varepsilon}^{\prime}=W_{1}\left(E^{\prime}\right)
$$

Thus the equivalence (i) $\Leftrightarrow$ (ii) has been established.

Next we turn to the equivalence (ii) $\Leftrightarrow$ (iii). First we derive equivalence of (6.5) and (6.8). That (6.5) implies (6.8) follows from substitution of CPT. So we assume (6.8) and derive (6.5). Suppose $W_{1}(A \cup B) \leq W_{1}(S-E)$ and

$$
W_{1}(C)=\pi_{1}(B, A) \text {. }
$$

We show that $W_{2}(C) \geq \pi_{2}(B, A)$.

Case 1: $W_{1}(A \cup B)<1$. Because $W_{1}$ and $W_{2}$ order events the same way, $W_{1}(F \cup G)=W_{1}(G)$ if and only if $W_{2}(F \cup G)=W_{2}(G)$, so a decision weight $\pi_{1}(F, G)=0$ if and only if $\pi_{2}(F, G)=0$. This holds in particular for $G=\varnothing$, i.e., $W_{1}(F)=0$ if and only if $W_{2}(F)=0$. Hence, in view of the assumed $W_{1}(C)=\pi_{1}(B, A)$, either all of $W_{1}(C), \pi_{1}(B, A), W_{2}(C), \pi_{2}(B, A)$ are 0 , or none. If they are all 0 , then $W_{2}(C)=\pi_{2}(B, A)$ and we are done. So assume, from now on:

$$
W_{1}(C), \pi_{1}(B, A), W_{2}(C), \pi_{2}(B, A) \text { are all positive. }
$$

If $W_{1}(A)=0$ then also $W_{2}(A)=0$, further by (6.5) then $W_{1}(C)=W_{1}(A \cup B)$ which implies $W_{2}(C)=$ $W_{2}(A \cup B)$; from these equalities the consequent inequality in (6.5) follows as an equality. Therefore assume also that

$$
W_{1}(A)>0 \text {. }
$$

${ }^{16}$ For this compatibility of boundary constraints it is essential that the boundary constants $\varepsilon$ and $\varepsilon^{\prime}$ for upper and lower SA can be different, and that similarly the boundary events $E$ and $E^{\prime}$ can be different. 
Since also $W_{1}(A \cup B)<1,0<x<X$ can be found such that

$$
x \sim_{1}(0, S-(A \cup B) ; x, B ; X, A) .
$$

Then also

$$
(x, S-C ; X, C) \sim_{1}(X, A \cup B)
$$

by the antecedent equality in (6.5), because the CPT difference between the left prospects is the same as between the right, i.e.,

$$
W_{1}(C)\left(v_{1}(X)-v_{1}(x)\right)=\left(W_{1}(A \cup B)-W_{1}(A)\right)\left(v_{1}(X)-v_{1}(x)\right) .
$$

Now $W_{2}(A)>0$ and $W_{2}(A \cup B)<W_{2}(S)=1$ follow from the corresponding inequalities for $W_{1}$; therefore $0<y<Y$ can be found such that

$$
y \sim_{2}(0, S-(A \cup B) ; y, B ; Y, A) .
$$

Condition (6.8) implies

$$
(y, S-C ; Y, C) \succcurlyeq_{2}(Y, A \cup B) .
$$

Comparing the CPT difference of the left prospects to that of the right prospects, we get $W_{2}(C)\left(v_{2}(Y)-v_{2}(y)\right) \geq\left(W_{2}(A \cup B)-W_{2}(A)\right)\left(v_{2}(Y)-v_{2}(y)\right)$, i.e., $W_{2}(C) \geq W_{2}(A \cup B)-W_{2}(A)$; (6.5) has been demonstrated.

Case 2: $W_{1}(A \cup B)=1$. If also $W_{1}(A)=1$, then $W_{2}(A \cup B)=W_{2}(S)=1=W_{2}(A)$ follows, implying (6.5). Hence assume $W_{1}(A)<1$. By solvability we can find, for $n$ sufficiently large, events $B_{n}$ such that $B_{n} \subset B$ and $W_{1}\left(A \cup B_{n}\right)=1-1 / n>W_{1}(A)$, and (recall that $W_{1}(C)=W_{1}(A \cup B)-W(A)$ $>0)$ events $C_{n} \subset C$ such that $W_{1}\left(C_{n}\right)=W_{1}(C)-1 / n$. Then $W_{1}\left(C_{n}\right)=W_{1}\left(A \cup B_{n}\right)-W_{1}(A)$. By Case $1, W_{2}\left(C_{n}\right) \geq W_{2}\left(A \cup B_{n}\right)-W_{2}(A)$. By continuity of $\phi, W_{2}\left(C_{n}\right)$ tends to $W_{2}(C)$, and $W_{2}\left(A \cup B_{n}\right)$ tends to $W_{2}(A \cup B)$, hence the inequality $W_{2}(C) \geq W_{2}(A \cup B)-W_{2}(A)$ follows: (6.5) has been proved.

Next we turn to the equivalence (6.6) $\Leftrightarrow(6.9)$. That (6.6) implies (6.9) follows from substitution of CPT. So we assume (6.9) and derive (6.6). Suppose $W_{1}(A) \geq W_{1}\left(E^{\prime}\right)$ and

$$
\pi_{1}(C, S-C)=\pi_{1}(B, A) .
$$

We show that $\pi_{2}(C, S-C) \geq \pi_{2}(B, A)$.

Case 1: $W_{1}(A)>0$. Because $W_{1}$ and $W_{2}$ order events the same way, and $\pi_{1}(C, S-C)=\pi_{1}(B, A)$, either all of $\pi_{1}(C, S-C), \pi_{1}(B, A), \pi_{2}(C, S-C), \pi_{2}(B, A)$ are 0 , or none. If they are all 0 , then $\pi_{2}(C, S-C)=\pi_{2}(B, A)$ and we are done. So suppose, from now on:

$$
\pi_{1}(C, S-C), \pi_{1}(B, A), \pi_{2}(C, S-C), \pi_{2}(B, A) \text { are all positive. }
$$

By monotonicity of capacities, $W_{1}(S-C) \geq W_{1}(A)$. Now, by the antecedent equality in (6.6), $W_{1}(S-C)=W_{1}(A)$ if and only if $W_{1}(A \cup B)=1=W_{1}(S)$; because $W_{1}$ and $W_{2}$ order events the same way, similar equalities then hold for $W_{2}$, which implies the consequent inequality in (6.6). Therefore we assume that

$$
W_{1}(S-C)>W_{1}(A) .
$$

Since also $W_{1}(A)>0,0<x<X$ can be found such that

$$
(x, S-C) \sim_{1}(X, A) .
$$

Then also

$$
x \sim_{1}(0, S-(A \cup B) ; x, B ; X, A),
$$

by the antecedent equality in (6.6), because the CPT difference between the left prospects is the same as between the right, i.e.,

$$
\left[1-W_{1}(S-C)\right] v_{1}(x)=\left[W_{1}(A \cup B)-W_{1}(A)\right] v_{1}(x) .
$$

The inequalities $W_{2}(S-C)>W_{2}(A)>0$ follow from the corresponding inequalities for $W_{1}$; therefore $0<y<Y$ can be found such that

$$
(y, S-C) \sim_{2}(Y, A) .
$$


Now (6.9) implies

$$
y \succcurlyeq_{2}(0, S-(A \cup B) ; y, B ; Y, A) .
$$

This and the $\sim_{2}$ indifference imply, by taking CPT differences between left and right prospects and dividing by $v_{2}(y)$, that $1-W_{2}(S-C) \geq W_{2}(A \cup B)-W_{2}(A)$. (6.6) has been demonstrated.

Case 2: $W_{1}(A)=0$. If $W_{1}(A \cup B)=0$, then $W_{2}(A \cup B)=0$, and (6.6) follows. Suppose therefore that $W_{1}(A \cup B)>0$. By solvability we can find, for $n$ sufficiently large, $A_{n}$ such that $A \subset A_{n} \subset A \cup B$ and $W_{1}\left(A_{n}\right)=1 / n$, and next (noting that $1-W_{1}(S-C)=W_{1}(A \cup B)-W_{1}(A)>0$, hence $W_{1}(S-$ $C)<1)$ we can find $C_{n} \subset C$ such that $W_{1}\left(S-C_{n}\right)=W_{1}(S-C)+1 / n$. Setting $B_{n}=(A \cup B)-A_{n}$, we get $1-W_{1}\left(S-C_{n}\right)=W_{1}\left(A_{n} \cup B_{n}\right)-W_{1}\left(A_{n}\right)$. By Case $1,1-W_{2}\left(S-C_{n}\right) \geq W_{2}\left(A_{n} \cup B_{n}\right)-$ $W_{2}\left(A_{n}\right)$ follows, i.e., $1-W_{2}\left(S-C_{n}\right) \geq W_{2}(A \cup B)-W_{2}\left(A_{n}\right)$. This inequality implies, by continuity of $\phi, 1-W_{2}(S-C) \geq W_{2}(A \cup B)-W_{2}(A)$, and (6.6) has been established.

Q.E.D.

\section{APPENDIX B: REFLECTION}

This section presents preference conditions for reflection under risk, i.e., $w^{+}=w^{-}$.

Proposition B1: Reflection is satisfied if and only if $\succcurlyeq$ satisfies the following condition:

$$
\begin{aligned}
\text { If } x & \sim(0,1-p-q ; x, q ; X, p) \text { and } \\
(x, 1-r ; X, r) & \sim(X, p+q), \text { then } \\
-y & \sim(-Y, p ;-y, q ; 0,1-p-q) \text { implies } \\
(-Y, r ;-y, 1-r) & \sim(-Y, p+q),
\end{aligned}
$$

for all $-Y<-y<0<x<X$, and $p, q, r \in] 0,1[$.

Proof: First we demonstrate necessity of (B1). The first two indifferences in (B1) imply that $w^{+}(r)(v(X)-v(x))=\left(w^{+}(p+q)-w^{+}(p)\right)(v(X)-v(x))$, i.e., $w^{+}(r)=w^{+}(p+q)-w^{+}(p)$. By reflection then $w^{-}(r)=w^{-}(p+q)-w^{-}(p)$. This implies $w^{-}(r)(v(-y)-v(-Y))=\left(w^{-}(p+q)-\right.$ $\left.w^{-}(p)\right)(v(-y)-v(-Y))$; the left-hand side is the CPT difference between the lower pair of prospects left of $\sim$, the right-hand side is the CPT difference between the lower pair of right prospects. Therefore the third indifference in (B1) implies the fourth. We conclude that reflection implies (B1).

Next we assume that (B1) holds, and derive reflection. Take $p_{0}, \ldots, p_{n}$ such that $w^{+}\left(p_{j}\right)=j / n$ for all $j$. Then take, for all $2 \leq j \leq n-1, X^{j}>x^{j}>0$ such that

$$
x^{j} \sim\left(0,1-p_{j} ; x^{j}, p_{j}-p_{j-1} ; X^{j}, p_{j-1}\right) .
$$

The following indifference is obtained by substitution of CPT, where the CPT value of both prospects is increased by $\left(v\left(X^{j}\right)-v\left(x^{j}\right)\right) / n$ :

$$
\left(x^{j}, 1-p_{1} ; X^{j}, p_{1}\right) \sim\left(X^{j}, p_{j}\right) .
$$

Next take, for all $2 \leq j \leq n-1,-Y^{j}<-y^{j}<0$ such that

$$
-y^{j} \sim\left(-Y^{j}, p_{j-1} ;-y^{j}, p_{j}-p_{j-1} ; 0,1-p_{j}\right) .
$$

It follows from the above three indifferences and (B1) that

$$
\left(-Y^{j}, p_{1} ;-y^{j}, 1-p_{1}\right) \sim\left(-Y^{j}, p_{j}\right) .
$$

The two loss-indifferences, and CPT, imply that $w^{-}\left(p_{1}\right)=w^{-}\left(p_{j}\right)-w^{-}\left(p_{j-1}\right)$. As this holds for all $2 \leq j \leq n-1, w^{-}\left(p_{j}\right)-w^{-}\left(p_{j-1}\right)=w^{-}\left(p_{1}\right)$ for all such $j$. So, for a positive constant $\lambda, w^{-}=\lambda w^{+}$ on $\left\{p_{1}, \ldots, p_{n-1}\right\}$, which set is the inverse under $w^{+}$of $\{1 / n, \ldots, 1-1 / n\}$. It follows that $\lambda$ is independent of $n$ (for $k \neq m$, compare to $i=k \cdot m$ ). As $n$ tends to infinity it follows, firstly, that $p_{n-1}$ tends to 1 , next, that $\lambda$ must be 1 , then, finally, that the continuous strictly increasing functions $w^{+}$ and $w^{-}$must be identical. This establishes reflection.

Q.E.D. 
Because the weighting function is $S$-shaped, it is similar to its dual; hence the qualitative predictions of CPT and rank-dependent utility are not very different. The key difference is that reflection implies $w^{+}(1 / 2)<1 / 2 \Leftrightarrow w^{-}(1 / 2)<1 / 2$, whereas rank-dependent utility implies $w^{+}(1 / 2)<1 / 2 \Leftrightarrow w^{-}(1 / 2)>1 / 2$. In the study of Tversky and Kahneman (1992), the estimated weighting functions for all 25 subjects satisfied the inequalities $w^{+}(1 / 2)<1 / 2$ and $w^{-}(1 / 2)<1 / 2$, in accord with CPT.

Much less is known about the relation between the weighting functions for gains and losses in the context of uncertainty. We suspect that the close correspondence observed in the domain of risk may not always hold for uncertainty.

\section{REFERENCES}

AllaIs, M. (1988): “The General Theory of Random Choices in Relation to the Invariant Cardinal Utility Function and the Specific Probability Function," in Risk, Decision and Rationality, ed. by B. R. Munier. Dordrecht: Reidel, pp. 233-289.

ARrow, K. J. (1965): Aspects of the Theory of Risk-Bearing. Helsinki: Academic Bookstore. Elaborated as Arrow, K. J. (1971), Essays in the Theory of Risk-Bearing. Amsterdam: North-Holland.

BeLL, D.E. (1982): "Regret in Decision Making under Uncertainty," Operations Research, 30, 961-981.

Birnbaum, M. H., G. Coffey, B. A. Mellers, And R. Weiss (1992): "Utility Measurement: Configural-Weight Theory and the Judge's Point of View," Journal of Experimental Psychology: Human Perception and Performance, 18, 331-346.

CAMERER, C. F. (1992): "Recent Tests of Generalizations of Expected Utility Theory," in Utility Theories: Measurement and Applications, ed. by W. Edwards. Dordrecht: Kluwer Academic Publishers, pp. 207-251.

—_ (1994): "Individual Decision Making," in Handbook of Experimental Economics, ed. by J. H. Kagel and A. E. Roth. Princeton, NJ: Princeton University Press, forthcoming.

CAMERER, C., AND T. Ho (1994): "Violations of the Betweenness Axiom and Nonlinearity in Probability," Journal of Risk and Uncertainty, 8, 167-196.

CAMERER, C. F., AND M. Weber (1992): "Recent Developments in Modelling Preferences: Uncertainty and Ambiguity," Journal of Risk and Uncertainty, 5, 325-370.

Chateauneuf, A. (1991): "On the Use of Capacities in Modeling Uncertainty Aversion and Risk Aversion," Journal of Mathematical Economics, 20, 343-369.

Chateauneuf, A., AND M. Cohen (1994): "Risk Seeking with Diminishing Marginal Utility in a Non-Expected Utility Model," Journal of Risk and Uncertainty, 9, 77-91.

CHEw, S. H. (1989): “The Rank-Dependent Quasilinear Mean,” unpublished manuscript, Department of Economics, University of California, Irvine.

Chew, S. H., E. KARNI, AND Z. SAFra (1987): "Risk Aversion in the Theory of Expected Utility with Rank Dependent Probabilities," Journal of Economic Theory, 42, 370-381.

CoHEN, M., AND J. Y. JAFFRAY (1988): "Certainty Effect versus Probability Distortion: An Experimental Analysis of Decision Making under Risk," Journal of Experimental Psychology: Human Perception and Performance, 14, 554-560.

CoHEN, M., J. Y. JAFFRAY, AND T. SAID (1987): "Experimental Comparisons of Individual Behavior under Risk and under Uncertainty for Gains and for Losses," Organizational Behavior and Human Decision Processes, 39, 1-22.

ELLSBERG, D. (1961): "Risk, Ambiguity and the Savage Axioms," Quarterly Journal of Economics, 75, 643-669.

FishbuRn, P. C. (1982): “Nontransitive Measurable Utility,” Journal of Mathematical Psychology, 26, 31-67.

Fishburn, P. C., AND G. A. Kochenberger (1979): “Two-Piece von Neumann-Morgenstern Utility Functions," Decision Sciences, 10, 503-518.

Friedman, M., AND L. J. SAVAGE (1948): “The Utility Analysis of Choices Involving Risk," Journal of Political Economy, 56, 279-304.

GilboA, I. (1987): "Expected Utility with Purely Subjective Non-Additive Probabilities," Journal of Mathematical Economics, 16, 65-88. 
Gonzales, R. (1993): "New Experiments on the Probability Weighting Function,” presented at the Society for Mathematical Psychology Meetings, Norman, OK.

Heath, C., AND A. Tversky (1991): "Preference and Belief: Ambiguity and Competence in Choice under Uncertainty," Journal of Risk and Uncertainty, 4, 5-28.

Hershey, J. C. , AND P. H. J. Schoemaker (1980): "Prospect Theory's Reflection Hypothesis: A Critical Examination," Organizational Behavior and Human Performance, 25, 395-418.

HogarTh, R. M., AND H. J. EINHORN (1990): "Venture Theory: A Model of Decision Weights," Management Science, 36, 780-803.

KaChelmeier, S. J., AND M. Shehata (1992): "Examining Risk Preferences under High Monetary Incentives: Experimental Evidence from the People's Republic of China," American Economic Review, 82, 1120-1141; for comment see American Economic Review, 84, 1104-1106.

Kahneman, D., AND A. TVersky (1979): "Prospect Theory: An Analysis of Decision under Risk," Econometrica, 47, 263-291.

KARMARKAR, U. S. (1978): "Subjectively Weighted Utility: A Descriptive Extension of the Expected Utility Model," Organizational Behavior and Human Performance, 21, 61-72.

KARNI, E., ANd Z. SAFrA (1990): "Rank-Dependent Probabilities," Economic Journal, 100, 487-495.

KeYnes, J. M. (1921): A Treatise on Probability. London: McMillan. Second edition, 1948.

Knight, F. H. (1921): Risk, Uncertainty, and Profit. New York: Houghton Mifflin.

LATtimore, P. M., J. R. BAKer, AND A. D. WiTte (1992): "The Influence of Probability on Risky Choice," Journal of Economic Behavior and Organization, 17, 377-400.

Loomes, G., AND R. Sugden (1982): "Regret Theory: An Alternative Theory of Rational Choice under Uncertainty," Economic Journal, 92, 805-824.

LuCE, R. D, AND P. C. FishbuRn (1991): "Rank- and Sign-Dependent Linear Utility Models for Finite First-Order Gambles," Journal of Risk and Uncertainty, 4, 29-59.

Markowitz, H. (1952): “The Utility of Wealth," Journal of Political Economy, 60, 151-158.

PAYne, J. W., D. J. LAughhunN, AND R. CRUM (1981): “Aspiration Level Effects in Risky Behavior," Management Science, 27, 953-958.

Pratt, J. W. (1964): "Risk Aversion in the Small and in the Large," Econometrica, 32, 122-136.

Quiggin, J. (1982): “A Theory of Anticipated Utility," Journal of Economic Behaviour and Organization, 3, 323-343.

SCHMEIDLER, D. (1989): "Subjective Probability and Expected Utility without Additivity," Econometrica, 57, 571-587.

Segal, U. (1987): "The Ellsberg Paradox and Risk Aversion: An Anticipated Utility Approach," International Economic Review, 28, 175-202.

StARMER, C., AND R. Sugden (1989): "Violations of the Independence Axiom in Common Ratio Problems: An Experimental Test of Some Competing Hypotheses," Annals of Operations Research, 19, 79-101.

TVErSKy, A., AND C. Fox (1994): “Weighing Risk and Uncertainty,” Psychological Review, forthcoming.

TVERSKY, A., AND D. KAHNEMAN (1992): "Advances in Prospect Theory: Cumulative Representation of Uncertainty," Journal of Risk and Uncertainty, 5, 297-323.

WAKkeR, P. P. (1994): "Separating Marginal Utility and Probabilistic Risk Aversion," Theory and Decision, 36, 1-44.

Wakker, P. P., I. ERev, AND E. Weber (1994): “Comonotonic Independence: The Critical Test between Classical and Rank-Dependent Utility Theories," Journal of Risk and Uncertainty, 9, 195-230.

WAKkeR, P. P., AND A. TVERSky (1993): "An Axiomatization of Cumulative Prospect Theory," Journal of Risk and Uncertainty, 7, 147-176.

WeHrung, D. A. (1989): "Risk Taking over Gains and Losses: A Study of Oil Executives," Annals of Operations Research, 19, 115-139.

Wu, G., AND R. Gonzales (1994): "Curvature of the Probability Weighting Function," Harvard Business School, Boston, MA.

YAARI, M. E. (1987): “The Dual Theory of Choice under Risk," Econometrica, 55, 95-115. 